Article

\title{
Dietary Patterns Independent of Fast Food Are Associated with Obesity among Korean Adults: Korea National Health and Nutrition Examination Survey 2010-2014
}

\author{
Do-Yeon Kim ${ }^{1}\left(\right.$, Ahleum Ahn ${ }^{2}$, Hansongyi Lee ${ }^{1}\left(\right.$, Jaekyung Choi ${ }^{2, *}$ and Hyunjung Lim ${ }^{1,3, * \mathbb{C}}$ \\ 1 Research Institute of Medical Nutrition, Kyung Hee University, Seoul 02447, Korea; \\ peacedkim@gmail.com (D.-Y.K.); flowerlhsy@hanmail.net (H.L.) \\ 2 Department of Family Medicine, Research Institute of Medical Science, Konkuk University School of \\ Medicine, Seoul 05030, Korea; arumi81@naver.com \\ 3 Department of Medical Nutrition, Graduate School of East-West Medical Science, Kyung Hee University, \\ Yong-in 17104, Korea \\ * Correspondence: 20060051@kuh.ac.kr (J.C.); hjlim@khu.ac.kr (H.L.); \\ Tel.: +82-2-2030-7683 (J.C.); +82-31-201-2343 (H.L.)
}

Received: 10 September 2019; Accepted: 7 November 2019; Published: 12 November 2019

\begin{abstract}
Few studies have examined the multifaceted aspects of fast food consumption and dietary patterns for their effects on obesity. We examined the independent associations of obesity with fast food consumption and dietary pattern in Korean adults using a nationally representative cross-sectional survey. A total of 19,017 adults aged 19-64 years participated in the Korea National Health and Nutrition Examination Survey (KNHANES) 2010-2014. Fast food items were removed from diet and then dietary patterns were generated. Multivariate logistic regression analysis was used to examine the odds of overweight/obesity and central obesity according to fast food consumption and dietary patterns. Fast food consumers were about $10 \%$ of Korean adults. Both the "White rice and kimchi" pattern and "Meat and alcohol" pattern were associated with low intakes of fiber, calcium, vitamin $C$, grains, fruit, and milk $(p<0.05)$. Fast food consumers had higher "Meat and alcohol" and "Grains, fruit, and milk" patterns, and they had a lower "White rice and kimchi" pattern than non-fast food-consumers. Fast food consumers were not associated with overweight/obesity, whereas participants with the "Meat and alcohol" pattern had 14\% higher overweight/obesity (95\% CI: 1.01, 1.28 ) and $16 \%$ higher central obesity (95\% CI: 1.00, 1.34). Fast food consumption was not directly associated with obesity, whereas the "Meat and alcohol" pattern had independent associations with overweight/obesity and central obesity among Korean adults.
\end{abstract}

Keywords: adults; dietary pattern; fast food; KNHANES; obesity

\section{Introduction}

Obesity is a predisposing factor for several chronic diseases, such as hypertension, type 2 diabetes, cardiovascular disease, and some cancers [1]. The prevalence of obesity has nearly tripled (men: $3.2 \%$ to $10.8 \%$, women: $6.4 \%$ to $14.9 \%$ ) worldwide from 1975 to 2014 [2]. In many lower- and middle-income countries in Asia, including South Korea [3], the rate of adult obesity has increased as a negative consequence of increasing economic development, supposedly due to a "nutrition transition." For example, the traditional dietary pattern high in grains, vegetables, and oilseeds is being replaced by a Western pattern high in fats, caloric sweeteners, and animal-sourced foods [4,5]. In line with that trend, fast food consumption doubled in South Korea from 1998 to 2009 [6]. 
Several researchers are concerned about the increasing consumption of fast food [7], which is characterized by high energy density [8], excessive portion sizes, high glycemic load [9], and low intakes of vegetables, fruits, and milk [10]. Some studies suggest an association between fast food intake and poor health outcomes $[9,11,12]$. On the other hand, others reported that are no significant associations between fast food intake and health outcomes, such as obesity $[7,13,14]$. These inconsistent results may be because other factors that correlate with fast food consumption have been limited to assess dietary factors, such as access to fast food restaurants [15], dietary preferences, and poor eating behaviors [16], including dietary patterns, rather than fast food consumption itself [10,14].

A study tested the independent association of obesity with dietary patterns and fast food items by collecting detailed food intake data among children in the US [10], but to our knowledge, no study exists for adults. In Asian countries, many studies have been conducted to investigate the association between dietary pattern and obesity $[5,17,18]$, however, no study has examined for an independent association of weight gain with dietary pattern and fast food consumption.

To fill this gap, we examine the independent associations of overweight/obesity with dietary pattern and fast food consumption in Korean adults using nationally representative cross-sectional survey data.

\section{Materials and Methods}

\subsection{Participants and Exclusion Criteria}

We used data from the fifth and sixth Korea National Health and Nutrition Examination Survey (KNHANES) in 2010 and 2014. The KNHANES is a nationally representative, cross-sectional survey of the South Korean population that uses a clustered, multistage, stratified, and rolling sampling design to represent non-institutionalized civilian Koreans [19]. The KNHANES V-VI protocols were approved by the Institutional Review Board of the Korean Center for Disease Control and Prevention (IRB No. 2010-02CON-21-C, 2011-02CON-06-C, 2012-01EXP-01-2C, 2013-07CON-03-4C, 2013-12EXP-03-5C).

We merged the 2010-2014 KNHANES data on a total of 41,102 participants and then sequentially excluded participants with extreme energy intake ( $<500 \mathrm{kcal} /$ day or $>5000 \mathrm{kcal} /$ day) $(n=4744)$ [20]; who were pregnant $(n=133)$; who were $<19$ years or $\geq 65$ years $(n=15,824)$; who were missing height or weight measurements $(n=62)$; or who had missing weight values $(n=1322)$. After applying these exclusion criteria, the total number of participants for this analysis was 19,017 (7516 men and 11,563 women).

\subsection{Anthropometric Measurements}

Height (Seca 225; SECA, Hamburg, Germany), weight (GL-6000-20; CASKOREA Co., Ltd., Seoul, Korea), and waist circumference (Seca 200; SECA, Hamburg, Germany) were obtained using standardized techniques and calibrated equipment. Body mass index (BMI) was calculated from these measurements as weight $(\mathrm{kg})$ divided by height squared $\left(\mathrm{m}^{2}\right)$. Overweight (BMI of $23-25 \mathrm{~kg} / \mathrm{m}^{2}$ ) and obesity (BMI $\geq 25 \mathrm{~kg} / \mathrm{m}^{2}$ ) were defined based on World Health Organization recommendations for Asians [21].

\subsection{Dietary Assessment and Fast Food Consumption}

Dietary data were assessed by trained dietary interviewers using the single 24-h recall method in the homes of the participants one week after the health interview and health examination. Energy and nutrient intakes for each subject were calculated using the Korean Foods and Nutrients Database of the Rural Development Administration [22,23], which contains 3095 food items.

Fast food consumption was defined as the intake of any of 48 Western fast food items (e.g., hamburgers, French fries, pizza, onion rings, fried chicken, sandwiches, apple pie, and cheese sticks). We classified adults as fast food consumers (FF consumers) if they consumed at least one food sourced 
from fast food on their day's recall and non-fast food consumers (non-consumers) if they did not consume any fast food.

\subsection{Food Grouping}

We divided food data into 25 food groups from the 18 food groups in the Korean Nutrient Database [24] to simplify the interpretation of components. Intake of grains and their products is very high in the Korean population; therefore, we subdivided this food group into four subgroups based on the nutrient profile: white rice, other grains, flour and bread, and noodles. Kimchi (traditional fermented cabbage) was also placed in a group separate from the vegetable group because it is a traditional side dish in Korea. The beverage group was disaggregated into soda, sugar-sweetened beverages (SSBs), and non-sugar beverages based on sugar content [25].

\subsection{Dietary Pattern Analysis Excluding Fast Food}

To identify dietary patterns excluding fast food, all fast food items were removed from the 25 food groups. Because of the non-normal distribution of data, we transformed the percentage of total energy contribution for each food group per day and then dietary patterns were generated by a K-means cluster analysis using SAS FASTCLUS (SAS Institute, Cary, NC, USA). This K-means procedure generates clusters by comparing the Euclidean distance between each subject and each cluster center [26]. Two to five solutions were examined to assess which set of clusters were more meaningful in determining dietary patterns. In the end, three clusters were identified as distinct patterns and labeled with descriptive names.

Almost half of the subjects ( $48 \%$ ) were assigned to the "White rice and kimchi" dietary pattern, in which consumption of white rice accounted for about $50 \%$ of total energy intake, and consumption of kimchi was higher than in the other two groups. A total of $40 \%$ of the subjects were assigned to the Grain, fruit, and milk dietary pattern, which was characterized by a variety of food groups, such as whole grains, bread, noodles, potatoes, nuts, fruit, eggs, milk, and oils. The remaining $12 \%$ of subjects were assigned to the "Meat and alcohol" dietary pattern based on their predominant food groups (Supplementary Table S1).

\subsection{Covariates}

Information on socio-demographic characteristics and health-related behaviors were obtained using in-person interviews by a trained researcher. Household income was divided into quartiles. Education level was categorized into three groups: $\leq$ middle school, high school, and $\geq$ college degree. Region was divided into two groups: urban and rural. Smoking status was divided into three groups: non-smokers, former smokers, and current smokers. Physical activity was assessed using the short form of International Physical Activity Questionnaire (IPAQ) [27]. The IPAQ suggests the level of physical activity by a Metabolic Equivalent Task (MET): vigorous MET-minutes/week $=8.0 \times$ minutes of activity per day $\times$ days of activity per week, moderate MET-minutes/week $=4.0 \times$ minutes of activity per day $\times$ days of activity per week, and walking MET-minutes/week $=3.3 \times$ minutes of activity per day $\times$ days of activity per week. Then, physical activity was divided into three groups: "Health-Enhancing" when the total score was 3000 MET-minutes/week or more, "Active" when the total score was 600 MET-minutes or more, and "Inactive" when the total score was below 600 MET-minutes [27]. Total energy intake was divided into age and sex-specific quintiles. Weight status was categorized into three groups: non-overweight, overweight, and obese.

\subsection{Statistical Analysis}

The analysis was performed using PROC SURVEY in SAS 9.4 (SAS Institute, Cary, NC, USA) to take into account the complex sampling design and appropriate sampling weights for the national survey. Two-sided chi-square and $t$-tests $(p<0.05)$ were used to compare sociodemographic characteristics according to fast food consumption and dietary pattern. We tested the associations between dietary 
pattern and dietary outcomes using the "Grain, fruit, and milk" pattern as reference. To estimate the associations between fast food consumption and each dietary pattern, we used multiple binary logistic regression models using the non-consumer as a reference. We adjusted for age, sex, household income, education, region, smoking, physical activity, total energy intake, and weight status.

Then, we used multivariate-adjusted logistic regression models to estimate the odds of overweight/obesity for fast food consumption and each dietary pattern after adjusting for age, sex, household income, education, total energy intake, smoking, and physical activity (model 1). Model 2 was adjusted for potential confounders and dietary pattern to determine the direct association between fast food consumption and weight status, and adjusted for confounders with fast food consumption to estimate the independent association between dietary pattern and weight status. Statistical significance was achieved when $p<0.05$.

\section{Results}

\subsection{General Characteristics}

General characteristics according to fast food consumption and dietary pattern are shown in Table 1. A total of $9.7 \%$ of adults were categorized into FF consumers. In general, FF consumers of all dietary patterns tended to be younger and more highly educated than non-consumers $(p<0.0001)$. According to dietary pattern, followers of the "Grain, fruit, eggs, and milk" pattern had a higher proportion of young, female, and highly educated individuals and fewer smokers regardless of fast food consumption $(p<0.0001)$. Those following the "White rice and kimchi pattern" were more likely to be older with low education and those following the "Meat and alcohol pattern" were more likely to be male and current smokers regardless of fast food consumption $(p<0.0001)$.

\subsection{Total Intake and Dietary Intake Excluding Fast Food}

The findings for total intake and dietary intake excluding fast food according to fast food consumption are shown in Table 2. A greater intake of meat and its products and soda and a lower intake of grain, legumes, nuts and seeds, vegetables, and fruit were observed among FF consumers not only for total intake $(10.6 \pm 0.2,2.4 \pm 0.1,3.3 \pm 0.2,1.4 \pm 0.1,0.6 \pm 0.1,2.4 \pm 0.1$, and $2.9 \pm 0.1 \mathrm{kcal} / \mathrm{day}$, respectively) but also for dietary intake excluding fast food $(12.9 \pm 0.3,3.3 \pm 0.2,4.0 \pm 0.2,1.7 \pm 0.1$, $0.8 \pm 0.1,2.8 \pm 0.1$, and $3.4 \pm 0.1 \mathrm{kcal} /$ day, respectively) than non-consumers $(p<0.05)$. In addition, $\mathrm{FF}$ consumers had higher total energy $(2741 \pm 30 \mathrm{kcal} /$ day $)$ and fat intake $(25.3 \pm 0.2 \%)$ than non-consumers $(2048 \pm 9 \mathrm{kcal} /$ day and $18.7 \pm 0.1 \%$, respectively; $p<0.05)$ in total intakes, and they also had higher total energy and fat intake even in dietary intake excluding fast food $(2230 \pm 26 \mathrm{kcal} /$ day and $22.5 \pm 0.2 \%$, respectively) than non-consumers (2048 $\pm 9 \mathrm{kcal} /$ day and $18.7 \pm 0.1 \%$, respectively; $p<0.05)$. 
Table 1. General characteristics according to fast food consumption and dietary patterns in adults when fast food items are excluded: KNHANES $2010-2014{ }^{1}$.

\begin{tabular}{|c|c|c|c|c|c|c|c|c|c|c|c|c|c|c|c|c|c|}
\hline & \multicolumn{4}{|c|}{ Grains, Fruit, and Milk Pattern ${ }^{2}$} & \multicolumn{5}{|c|}{ White Rice and Kimchi Pattern } & \multicolumn{5}{|c|}{ Meat and Alcohol Pattern } & \multirow[b]{3}{*}{$p$-Value ${ }^{4}$} & \multirow[b]{3}{*}{$p$-Value ${ }^{5}$} & \multirow[b]{3}{*}{$p$-Value } \\
\hline & \multirow{2}{*}{\multicolumn{2}{|c|}{$\begin{array}{c}\text { Non-Consumers }^{3} \\
(n=6541 ; 84.3 \%)\end{array}$}} & \multirow{2}{*}{\multicolumn{2}{|c|}{$\begin{array}{c}\text { FF Consumers } \\
(n=1085 ; \\
15.7 \%)\end{array}$}} & \multirow{3}{*}{$\begin{array}{c}p \text {-Value }{ }^{4} \\
<0.0001\end{array}$} & \multirow{2}{*}{\multicolumn{2}{|c|}{$\begin{array}{c}\text { Non-Consumers } \\
(n=8695 ; \\
94.5 \%)\end{array}$}} & \multirow{2}{*}{\multicolumn{2}{|c|}{$\begin{array}{l}\text { FF Consumers } \\
(n=454 ; 5.5 \%)\end{array}$}} & \multirow{3}{*}{$\begin{array}{c}p \text {-Value }^{4} \\
<0.0001\end{array}$} & \multirow{2}{*}{\multicolumn{2}{|c|}{$\begin{array}{c}\text { Non-Consumers } \\
(n=1937 ; \\
85.7 \%)\end{array}$}} & \multicolumn{2}{|c|}{ FF Consumers } & & & \\
\hline & & & & & & & & & & & & & & $\begin{array}{l}\text { 305; } \\
\%)\end{array}$ & & & \\
\hline & & & & & & & & & & & & & & & & & \\
\hline $19-39$ years & 48.0 & $(0.8)$ & 72.6 & $(1.6)$ & & 37.9 & $(0.8)$ & 59.6 & $(2.7)$ & & 43.0 & $(1.4)$ & 71.7 & $(2.9)$ & & & \\
\hline 40-64 years & 52.0 & $(0.8)$ & 27.4 & $(1.6)$ & & 62.1 & $(0.8)$ & 40.4 & $(2.7)$ & & 57.0 & $(1.4)$ & 28.3 & $(2.9)$ & & & \\
\hline Sex & & & & & 0.0546 & & & & & 0.7924 & & & & & 0.9789 & $<0.0001$ & $<0.0001$ \\
\hline Male & 40.3 & $(0.7)$ & 44.0 & $(1.7)$ & & 51.9 & $(0.6)$ & 52.6 & $(2.7)$ & & 73.9 & $(1.0)$ & 73.8 & $(2.6)$ & & & \\
\hline Female & 59.7 & $(0.7)$ & 56.0 & $(1.7)$ & & 48.1 & $(0.6)$ & 47.4 & $(2.7)$ & & 26.1 & $(1.0)$ & 26.2 & $(2.6)$ & & & \\
\hline Education & & & & & $<0.0001$ & & & & & $<0.0001$ & & & & & $<0.0001$ & $<0.0001$ & $<0.0001$ \\
\hline$\leq$ Middle school & 12.9 & $(0.6)$ & 3.5 & $(0.5)$ & & 23.6 & $(0.7)$ & 12.0 & $(1.7)$ & & 13.7 & $(0.9)$ & 5.2 & $(1.4)$ & & & \\
\hline High school & 30.7 & $(0.8)$ & 27.2 & $(1.6)$ & & 34.4 & $(0.7)$ & 26.5 & (2.6) & & 35.3 & (1.4) & 27.9 & (3.1) & & & \\
\hline$\geq$ College degree & 56.4 & $(0.9)$ & 69.2 & $(1.6)$ & & 42.0 & $(0.8)$ & 61.5 & $(3.0)$ & & 51.1 & $(1.5)$ & 66.9 & $(3.1)$ & & & \\
\hline Household income & & & & & 0.3162 & & & & & 0.2808 & & & & & 0.2902 & $<0.0001$ & 0.0554 \\
\hline Low (Q1) & 7.5 & $(0.5)$ & 9.3 & $(1.3)$ & & 11.9 & $(0.5)$ & 9.4 & $(1.9)$ & & 8.3 & $(0.8)$ & 7.5 & $(1.7)$ & & & \\
\hline Low middle (Q2) & 24.5 & $(0.8)$ & 22.5 & (1.7) & & 29.2 & $(0.7)$ & 27.4 & $(2.6)$ & & 25.7 & $(1.3)$ & 28.1 & (3.1) & & & \\
\hline Middle-High (Q3) & 32.0 & $(0.8)$ & 32.9 & (1.9) & & 31.1 & $(0.7)$ & 36.1 & $(2.8)$ & & 32.9 & (1.3) & 27.1 & $(3.0)$ & & & \\
\hline High (Q4) & 36.1 & $(1.0)$ & 35.2 & $(1.9)$ & & 27.8 & $(0.8)$ & 27.1 & $(2.6)$ & & 33.1 & $(1.4)$ & 37.3 & (3.3) & & & \\
\hline Region & & & & & 0.1189 & & & & & 0.0539 & & & & & 0.0240 & $<0.0001$ & 0.1463 \\
\hline Urban & 76.6 & $(1.1)$ & 79.4 & $(1.9)$ & & 68.4 & $(1.1)$ & 73.9 & $(2.7)$ & & 71.9 & (1.6) & 79.2 & $(2.9)$ & & & \\
\hline Rural & 23.4 & $(1.1)$ & 20.6 & $(1.9)$ & & 31.6 & $(1.1)$ & 26.1 & $(2.7)$ & & 28.1 & $(1.6)$ & 20.8 & $(2.9)$ & & & \\
\hline Smoking & & & & & 0.3475 & & & & & 0.5807 & & & & & 0.6760 & $<0.0001$ & $<0.0001$ \\
\hline Current & 20.1 & $(0.7)$ & 22.3 & $(1.5)$ & & 25.9 & $(0.6)$ & 24.1 & $(2.5)$ & & 45.8 & (1.4) & 48.7 & (3.4) & & & \\
\hline Past & 16.5 & $(0.6)$ & 17.0 & (1.5) & & 19.1 & $(0.5)$ & 17.7 & $(2.3)$ & & 24.0 & $(1.2)$ & 21.8 & $(2.7)$ & & & \\
\hline Never & 63.4 & $(0.7)$ & 60.7 & $(1.9)$ & & 55.1 & $(0.6)$ & 58.2 & (2.9) & & 30.1 & (1.2) & 29.5 & $(2.9)$ & & & \\
\hline Physical activity & & & & & & & & & & & & & & & & & \\
\hline Inactive & 35.5 & $(0.8)$ & 36.7 & $(1.8)$ & 0.4850 & 39.8 & $(0.7)$ & 39.7 & $(2.7)$ & 0.9953 & 33.2 & $(1.3)$ & 34.3 & $(3.2)$ & 0.1332 & $<0.0001$ & 0.7707 \\
\hline Active & 44.3 & $(0.8)$ & 42.0 & (1.8) & & 39.5 & $(0.6)$ & 39.8 & $(2.7)$ & & 39.1 & (1.4) & 44.1 & (3.3) & & & \\
\hline Health enhancing & 20.1 & $(0.6)$ & 21.3 & (1.5) & & 20.7 & $(0.6)$ & 20.6 & (2.4) & & 27.7 & $(1.2)$ & 21.6 & $(2.5)$ & & & \\
\hline
\end{tabular}

KNHANES, Korea national health and nutrition examination survey; $\mathrm{FF}$, fast food; non-consumer, non-fast food consumers. ${ }^{1}$ Values are \% (SE). Data were weighted to represent adults aged 19-64 years from KNHANES 2010-2014. ${ }^{2}$ Dietary patterns excluding fast food were determined by cluster analysis using dietary intake (kcal/day) minus any fast food items consumed. ${ }^{3}$ Fast food consumers are defined as those who consumed more than one fast food item, and non-consumers are defined as those who did not consume any fast food. ${ }^{4} p$-values for differences between FF consumers and non-consumers in each cluster using chi-square test for proportions and analysis of variance for means. ${ }^{5} p$-values for differences across clusters in non-consumers using chi-square test for proportions and analysis of variance for means. ${ }^{6} p$-values for differences across clusters in FF consumers using chi-square test for proportions and analysis of variance for means. 
Table 2. Total dietary intake and dietary intake excluding fast food items according to fast food consumption among adults: KNHANES 2010-2014 ${ }^{1}$.

\begin{tabular}{|c|c|c|c|c|}
\hline & \multicolumn{2}{|c|}{ Total Intake } & \multicolumn{2}{|c|}{ Intake Minus FF 2} \\
\hline & Non-Consumer ${ }^{3}$ & FF Consumer & Non-Consumer & FF Consumer \\
\hline & $(n=17,173 ; 90.3 \%)$ & $(n=1844 ; 9.7 \%)$ & $(n=17,173 ; 90.3 \%)$ & $(n=1844 ; 9.7 \%)$ \\
\hline \multicolumn{5}{|l|}{ Food group (\% of energy) 4} \\
\hline White rice & $31.90 \pm 0.21$ & $17.91 \pm 0.37^{*}$ & $31.90 \pm 0.21$ & $21.56 \pm 0.42 *$ \\
\hline Grains & $6.06 \pm 0.10$ & $3.34 \pm 0.15 *$ & $6.06 \pm 0.10$ & $3.98 \pm 0.18 *$ \\
\hline Sweets & $1.89 \pm 0.03$ & $1.57 \pm 0.07^{*}$ & $1.89 \pm 0.03$ & $1.90 \pm 0.09$ \\
\hline Legumes & $2.36 \pm 0.04$ & $1.43 \pm 0.07 *$ & $2.36 \pm 0.04$ & $1.73 \pm 0.09 *$ \\
\hline Nuts and seeds & $1.02 \pm 0.03$ & $0.64 \pm 0.06^{*}$ & $1.02 \pm 0.03$ & $0.76 \pm 0.07 *$ \\
\hline Vegetables & $3.16 \pm 0.03$ & $2.36 \pm 0.08 *$ & $3.16 \pm 0.03$ & $2.84 \pm 0.09 *$ \\
\hline Kimchi & $1.43 \pm 0.02$ & $0.81 \pm 0.03 *$ & $1.43 \pm 0.02$ & $0.98 \pm 0.03 *$ \\
\hline Fruit & $4.45 \pm 0.08$ & $2.89 \pm 0.12 *$ & $4.45 \pm 0.08$ & $3.44 \pm 0.14 *$ \\
\hline Meat and its products & $8.81 \pm 0.11$ & $10.63 \pm 0.23 *$ & $8.81 \pm 0.11$ & $12.93 \pm 0.28 *$ \\
\hline Eggs & $1.90 \pm 0.03$ & $1.69 \pm 0.06^{*}$ & $1.90 \pm 0.03$ & $2.04 \pm 0.08$ \\
\hline Fish and shellfish & $3.71 \pm 0.05$ & $2.41 \pm 0.10 *$ & $3.71 \pm 0.05$ & $2.91 \pm 0.13^{*}$ \\
\hline Milk and dairy products & $3.53 \pm 0.07$ & $3.46 \pm 0.14$ & $3.53 \pm 0.07$ & $4.42 \pm 0.21 *$ \\
\hline Oils & $3.27 \pm 0.03$ & $4.50 \pm 0.10 *$ & $3.27 \pm 0.03$ & $5.49 \pm 0.13^{*}$ \\
\hline Soda & $0.56 \pm 0.02$ & $2.40 \pm 0.11^{*}$ & $0.56 \pm 0.02$ & $3.30 \pm 0.16^{*}$ \\
\hline SSBs & $3.11 \pm 0.05$ & $2.44 \pm 0.11^{*}$ & $3.11 \pm 0.05$ & $3.00 \pm 0.15$ \\
\hline Alcohol & $4.22 \pm 0.11$ & $4.30 \pm 0.25$ & $4.22 \pm 0.11$ & $5.02 \pm 0.29 *$ \\
\hline Total energy, kcal/day & $2048.0 \pm 8.9$ & $2740.6 \pm 30.2^{*}$ & $2048.0 \pm 8.9$ & $2229.9 \pm 26.3^{*}$ \\
\hline $\begin{array}{c}\text { Total carbohydrate, } \\
\text { \% of energy }\end{array}$ & $63.6 \pm 0.2$ & $52.5 \pm 0.3 *$ & $63.6 \pm 0.2$ & $56.6 \pm 0.4 *$ \\
\hline Total fat, \% of energy & $18.7 \pm 0.1$ & $25.3 \pm 0.2 *$ & $18.7 \pm 0.1$ & $22.5 \pm 0.2 *$ \\
\hline
\end{tabular}

KNHANES, Korea national health and nutrition examination Survey; FF, fast food; non-consumer, non-fast food consumers. ${ }^{1}$ Values are mean \pm SE. Data were weighted to represent adults aged 19-64 years from KNHANES 2010-2014. * Statistically different from fast food non-consumers $\left(p<0.05\right.$ with $t$-test). ${ }^{2}$ Dietary intake minus fast food items. ${ }^{3}$ Fast food consumers are defined as those who consumed more than one fast food item, and non-consumers are defined as those who did not consume any fast food. ${ }^{4}$ Percentage of total energy intake from each food group in total dietary intake and dietary intake minus fast food according to fast food consumption.

\subsection{Dietary Intakes According to Dietary Pattern}

A multivariate regression model of each dietary intake with dietary pattern is shown in Table 3. The "White rice and kimchi" pattern and the "Meat and alcohol" pattern were associated with a lower consumption of healthy food and nutrient values than the "Grain, fruit, and milk" pattern. Participants following the "White rice and kimchi" pattern consumed significantly lower amounts of grains $(\beta=-60.5 ; 95 \% \mathrm{CI}:-68.9,-52.1 \mathrm{~g})$, milk and dairy products $(\beta=-42.3 ; 95 \% \mathrm{CI}:-47.5,-37.2 \mathrm{~g})$, fruit $(\beta=-33.0 ; 95 \%$ CI: $-38.2,-27.7 \mathrm{~g})$, oils $(\beta=-10.3 ; 95 \% \mathrm{CI}:-13.4,-7.1 \mathrm{~g})$, nuts and seeds $(\beta=-8.9$; $95 \%$ CI: $-11.8,-6.0 \mathrm{~g})$, and protein $(\beta=-5.4 ; 95 \% \mathrm{CI}:-6.4,-4.3 \mathrm{~g})$, fiber $(\beta=-0.6 ; 95 \% \mathrm{CI}:-0.8$, $-0.4 \mathrm{~g})$, calcium ( $\beta=-51.4 ; 95 \% \mathrm{CI}:-62.7,-40.1 \mathrm{~g})$, and vitamin $\mathrm{C}(\beta=-11.1 ; 95 \% \mathrm{CI}:-15.2,-7.0 \mathrm{~g})$ than those following the "Grain, fruit, and milk" pattern. For those following the "Meat and alcohol" pattern, the consumption of grains ( $\beta=-86.3 ; 95 \% \mathrm{CI}:-96.1,-76.5 \mathrm{~g})$, fruit $(\beta=-62.7 ; 95 \% \mathrm{CI}:-70.4$, $-54.9 \mathrm{~g})$, milk and dairy products $(\beta=-59.1 ; 95 \% \mathrm{CI}:-65.7,-52.4 \mathrm{~g})$, legumes $(\beta=-18.0 ; 95 \% \mathrm{CI}$ : $-22.7,-13.2 \mathrm{~g})$, nuts and seeds $(\beta=-6.3 ; 95 \% \mathrm{CI}:-10.4,-2.1 \mathrm{~g})$, eggs $(\beta=-11.8 ; 95 \% \mathrm{CI}:-15.6$, $-8.0 \mathrm{~g})$, oils $(\beta=-11.3 ; 95 \% \mathrm{CI}:-16.7,-5.8 \mathrm{~g})$, and fiber $(\beta=-1.7 ; 95 \% \mathrm{CI}:-2.0,-1.4 \mathrm{~g})$, calcium $(\beta=-113.1 ; 95 \% \mathrm{CI}:-130.5,-95.7 \mathrm{mg})$, vitamin $\mathrm{A}(\beta=-96.3 ; 95 \% \mathrm{CI}:-167.2,-25.4 \mu \mathrm{gRE})$, and vitamin $\mathrm{C}$ $(\beta=-33.2 ; 95 \%$ CI: $-39.7,-26.6 \mathrm{mg})$ was lower, although total energy intake ( $\beta=171.3 ; 95 \%$ CI: 137.8, $204.7 \mathrm{kcal})$ was higher than the "Grain, fruit, and milk" pattern. 
Table 3. Multiple linear regression analysis of the independent associations between dietary pattern minus fast food and dietary outcomes among adults: KNHANES 2010-2014 ${ }^{1}$.

\begin{tabular}{|c|c|c|}
\hline & \multicolumn{2}{|c|}{ Dietary Pattern ${ }^{2}$} \\
\hline & White Rice and Kimchi Pattern & Meat and Alcohol Pattern \\
\hline \multicolumn{3}{|l|}{ Food groups (kcal/day) } \\
\hline White rice & $505.4(494.8,516.1)$ & $36.8(21.4,52.2)$ \\
\hline Grains & $-60.5(-68.9,-52.1)$ & $-86.3(-96.1,-76.5)$ \\
\hline Legumes & $-3.2(-6.6,0.3)$ & $-18.0(-22.7,-13.2)$ \\
\hline Nuts and seeds & $-8.9(-11.8,-6.0)$ & $-6.3(-10.4,-2.1)$ \\
\hline Vegetables & $-1.0(-3.0,1.0)$ & $4.9(1.7,8.2)$ \\
\hline Kimchi & $5.0(3.8,6.1)$ & $-1.0(-2.7,0.6)$ \\
\hline Fruit & $-33.0(-38.2,-27.7)$ & $-62.7(-70.4,-54.9)$ \\
\hline Meat and its products & $-44.9(-53.2,-36.6)$ & $232.6(207.1,258.1)$ \\
\hline Eggs & $-1.5(-3.8,0.9)$ & $-11.8(-15.6,-8.0)$ \\
\hline Fish and shellfish & $9.1(4.5,13.6)$ & $18.6(10.1,27.2)$ \\
\hline Milk and dairy products & $-42.3(-47.5,-37.2)$ & $-59.1(-65.7,-52.4)$ \\
\hline Oils & $-10.3(-13.4,-7.1)$ & $-11.3(-16.7,-5.8)$ \\
\hline Soda & $-9.5(-11.9,-7.1)$ & $-12.4(-16.4,-8.4)$ \\
\hline SSBs & $-0.7(-4.3,3.0)$ & $-18.2(-23.6,-12.7)$ \\
\hline Alcohol & $-0.9(-5.0,3.2)$ & $594.7(572.2,617.2)$ \\
\hline \multicolumn{3}{|l|}{ Nutrients } \\
\hline Total energy (kcal/day) & $-62.8(-78.4,-47.1)$ & $171.3(137.8,204.7)$ \\
\hline Protein (g/day) & $-5.4(-6.4,-4.3)$ & $8.3(6.0,10.6)$ \\
\hline Fat (g/day) & $-13.0(-13.9,-12.1)$ & $0.6(-1.5,2.8)$ \\
\hline Carbohydrate (g/day) & $15.5(12.1,18.8)$ & $-102.7(-107.9,-97.4)$ \\
\hline Fiber (g/day) & $-0.6(-0.8,-0.4)$ & $-1.7(-2.0,-1.4)$ \\
\hline Calcium (mg/day) & $-51.4(-62.7,-40.1)$ & $-113.1(-130.5,-95.7)$ \\
\hline Vitamin A ( $\mu g R E /$ day $)$ & $-15.8(-58.9,27.2)$ & $-96.3(-167.2,-25.4)$ \\
\hline Vitamin C (mg/day) & $-11.1(-15.2,-7.0)$ & $-33.2(-39.7,-26.6)$ \\
\hline
\end{tabular}

KNHANES, Korea national health and nutrition examination survey; FF, fast food; non-consumer, non-fast food consumers. ${ }^{1}$ Data were weighted to represent adults aged 19-64 years from KNHANES 2010-2014. Values are $\beta$ coefficients ( $95 \%$ CIs) of dietary outcomes (food groups and nutrients), with dietary pattern as the independent variable using the "Grain, fruit, and milk" pattern as a reference. The model was adjusted for age, sex, income, education, region, smoking, total energy intake, and weight status. Bold indicates significance at $p$-value $<0.05$. 2 Dietary patterns minus fast food were determined by cluster analysis using dietary intake (kcal/day) excluding any fast food items consumed.

\subsection{Dietary Pattern According to Fast Food Consumption}

Adjusted OR and 95\% CI for the dietary pattern according to fast food consumption are shown in Table 4. The adjusted OR following the "Meat and alcohol" pattern increased by 1.2-fold (OR: 1.21; $95 \%$ CI: $1.02,1.43$ ) and following the "Grain, fruit, and milk" pattern increased by 2-fold (OR: 2.00; $95 \%$ CI: 1.76, 2.27) among FF consumer compared with non-consumers after adjusting for potential confounders. On the other hand, FF consumers were 59\% less likely than non-consumers to follow the "White rice and kimchi" pattern (OR: 0.41; 95\% CI: 0.35, 0.47).

\subsection{Overweight/obesity or Central Obesity According to Fast Food Consumption and Dietary Pattern}

Adjusted OR and 95\% CI for overweight/obesity or central obesity according to fast food consumption and dietary pattern are shown in Table 5. Although fast food consumption was not associated with overweight/obesity or central obesity after adjusting for covariates and dietary patterns, those who followed the "Meat and alcohol" pattern had a 14\% higher prevalence of overweight/obesity (OR: 1.14; 95\% CI: 1.04, 1.28) and a 16\% higher prevalence of central obesity (OR: 1.16; 95\% CI: 1.00, 1.34) than those who followed the "Grain, fruit, and milk" pattern after adjusting for covariates and fast food consumption. Furthermore, decreased odds of overweight/obesity were associated with 
the "White rice and kimchi" pattern (OR: 0.88; 95\% CI: 0.81, 0.95), although central obesity was not associated after controlling for covariates and fast food consumption.

Table 4. ORs (95\% CIs) for dietary pattern minus fast food items by fast food consumption among adults: KNHANES 2010-2014 ${ }^{1}$.

\begin{tabular}{|c|c|c|}
\hline & \multicolumn{2}{|c|}{ Fast Food Consumption $^{2}$} \\
\hline & Non-Consumer & FF Consumer \\
\hline & $(n=17,173 ; 90.3 \%)$ & $(n=1844 ; 9.7 \%)$ \\
\hline $\begin{array}{c}\text { Fast food intake (\% of energy) } \\
\text { Multivariable-adjusted OR }\end{array}$ & 0 & $13.27(0.03,84.13)$ \\
\hline \multicolumn{3}{|c|}{ Grain, fruit, and milk pattern } \\
\hline Model 14 & 1.0 (reference) & $2.08(1.83,2.36)$ \\
\hline Model 2 & 1.0 & $2.00(1.76,2.27)$ \\
\hline \multicolumn{3}{|c|}{ White rice and kimchi pattern } \\
\hline Model 1 & 1.0 & $0.37(0.32,0.42)$ \\
\hline Model 2 & 1.0 & $0.41(0.35,0.47)$ \\
\hline \multicolumn{3}{|c|}{ Meat and alcohol pattern } \\
\hline Model 1 & 1.0 & $1.45(1.23,1.71)$ \\
\hline Model 2 & 1.0 & $1.21(1.02,1.43)$ \\
\hline
\end{tabular}

KNHANES, Korea national health and nutrition examination Survey; FF, fast food; non-consumer, non-fast food consumers. ${ }^{1}$ Data were weighted to represent adults aged 19-64 years from KNHANES 2010-2014. Dietary patterns minus fast food were determined by cluster analysis using intake ( $\mathrm{kcal} /$ day) and excluding any fast food items consumed. ${ }^{2}$ Fast food consumers are defined as those who consumed more than one fast food item, and non-consumers are defined as those who did not consume any fast food. ${ }^{3}$ Values are medians (ranges). ${ }^{4}$ ORs (95\% CIs) for dietary pattern minus any fast food consumed by fast food consumption using multiple binary logistic regression models. Model 1 was adjusted for age, sex, income, education, region, physical activity, and smoking. Model 2 was adjusted for Model 1 plus total energy intake and weight status. Bold indicates significance at $p$-value $<0.05$.

Table 5. ORs (95\% CIs) for weight status with fast food consumption and dietary pattern minus any fast food items consumed among adults: KNHNES 2010-2014 ${ }^{1}$.

\begin{tabular}{|c|c|c|c|}
\hline & \multirow{2}{*}{ FF Consumption ${ }^{2}$} & \multicolumn{2}{|c|}{ Dietary Pattern $^{3}$} \\
\hline & & White Rice and Kimchi & Meat and Alcohol \\
\hline \multicolumn{4}{|l|}{ Overweight/obesity } \\
\hline Model $1^{4}$ & $1.04(0.92,1.17)$ & $0.88(0.82,0.96)$ & $1.14(1.01,1.28)$ \\
\hline Model 2 & $1.07(0.94,1.20)$ & $0.88(0.81,0.95)$ & $1.14(1.01,1.28)$ \\
\hline \multicolumn{4}{|l|}{ Central obesity } \\
\hline Model 1 & $1.15(0.97,1.36)$ & $1.09(0.99,1.20)$ & $1.16(1.01,1.35)$ \\
\hline Model 2 & $1.14(0.96,1.34)$ & $1.08(0.98,1.19)$ & $1.16(1.00,1.34)$ \\
\hline
\end{tabular}

KNHANES, Korea national health and nutrition examination Survey; FF, fast food; non-consumer, non-fast food-consumers. ${ }^{1}$ Data were weighted to represent adults aged 19-64 years from KNHANES 2010-2014. ${ }^{2}$ Fast food consumers are defined as those who consumed more than one fast food item, and non-consumers are defined as those who did not consume any fast food. ${ }^{3}$ Dietary patterns minus fast food were determined by cluster analysis using intake (kcal/day) and excluding any fast food items consumed. ${ }^{4}$ ORs (95\% CIs) for weight status (overweight/obesity and central obesity) with fast food consumption and dietary pattern minus fast food (reference: "Grain, fruit, and milk" pattern). Model 1 was adjusted for age, sex, income, education, region, smoking, total energy intake, and physical activity. Model 2 was adjusted for dietary pattern excluding fast food to find associations with fast food consumption and was adjusted for fast food consumption to find associations with dietary patterns excluding fast food. Bold indicates significance at $p$-value $<0.05$.

\section{Discussion}

This study contributes to the growing literature on unhealthy eating and overweight/obesity and is, to our knowledge, the first to examine independent associations among dietary patterns, fast food consumption, and overweight/obesity within a nationally representative sample of Korean adults. We found that the dietary pattern associated with health outcomes when fast food consumption is 
controlled, in particular the "Meat and alcohol" pattern, and that fast food consumption was not associated with weight gain when dietary patterns and other covariates were controlled.

Obesity results from a complex interaction of gender, environmental, genetic, and behavioral factors, and existing disease [28]. Among the behavioral factors, diet plays a major role in the development of overweight/obesity [4]. Previous research showed that people who consume fast food have higher energy and fat intakes, which are important contributors to overweight/obesity, suggesting that regulating the consumption of fast food might improve diets and health $[14,15,29]$. Our results also noted that FF consumers had higher total energy and fat intakes than non-consumers even when fast food items were excluded in the diet. Although the proportion of participants who consumed FF was low (10\%), but those were doubled over an 11-year period [6], and several Asian researchers are concerned about the increasing consumption of fast food because it has been suggested to be linked to obesity in Asia [7,13]. However, emerging evidence suggests that obesity is associated with an individual's dietary preferences and poor eating behavior, and sociodemographic characteristics, rather than being directly related to fast food $[6,16]$. In the crude model without adjustment in our results, fast food consumption was positively associated with overweight/obesity and central obesity (data not shown), but the significance disappeared after adjustment for potential confounders, which provides important information about the direct factors and prevalence of overweight/obesity.

Our results are of international significance because they contribute to an emerging evidence base that suggests that dietary pattern is independently associated with overweight/obesity regardless of fast food consumption [10], which was consistent with the result of subgroup analysis on non-consumer (data not shown). Furthermore, when we separated eating behavior into fast food consumption and dietary patterns with the fast food items removed, we were able to compare the probability of overweight/obesity for different eating behaviors at the same time. Our results are unique in showing that the "Meat and alcohol" pattern was positively associated with overweight/obesity, whereas the "White rice and kimchi" pattern showed an opposite association. Previous studies suggested that the consumption of red and processed meats was associated with the risk of obesity [30]. With respect to "White rice and kimchi" dietary pattern, a representative Korean study reported that a carbohydrate intake that composes more than $60 \%$ of total energy intake and the "White rice and kimchi" pattern had a lowering effect on BMI due to lower intakes of total fat. However, lowering HDL-cholesterol and increasing serum triglycerides are negative effects of the "White rice and kimchi" pattern; thus, even though that pattern is not positively associated with obesity, it does contribute to high intakes of carbohydrate and sodium and provides limited food composition [31]. In agreement, we found that people following the "White rice and kimchi" pattern derived $69 \%$ of their energy intake from carbohydrates (data not shown) and it may influence the lower prevalence of overweight/obesity.

In light of our findings, we suggest that dietary patterns are a more crucial factor in overweight/obesity than fast food consumption. Therefore, research is needed to determine what dietary patterns are healthy while excluding fast food consumption. Combining previous evidence that healthy patterns are characterized by high intakes of whole grains, fruits, and legumes and are rich in nutrients, such as antioxidants and associated with a reduction in metabolic risk factors [31,32], our results suggest that the "Grains, fruit, and milk" pattern could have preventive effects on weight gain through the combined influence of nutrients and food components compared to the "Meat and alcohol" pattern. Furthermore, for the first time, to our knowledge, we showed that adult Asian FF consumers were linked to the "Meat and alcohol" pattern or "Grain, fruit, and milk" pattern. Previous studies showed that FF consumers were associated with a Western dietary pattern but those analyses were based on US children [10]. Previous studies and our results thus suggest that those who consume the healthy "Grain, fruit, and milk" pattern are less likely to be overweight/obese than those who eat the "Meat and alcohol" pattern regardless of fast food consumption.

Our study has several limitations. First, dietary intake was estimated based on a 24-h dietary recall, which might not have been representative of a subject's typical intake. However, at the population level, it can provide rich details about mean dietary intake for a given day [33]. Second, this was a 
cross-sectional study; therefore, our study precludes inferences of causation, and the possibility of reverse causation remains.

Despite these limitations, the present study has many strengths. First, we used data from a nationally representative sample and took into account the complex sampling design effects to provide representative estimates. Second, we sufficiently analyzed a considerable range of available fast food items to identify FF consumers, and we separated fast food items from the rest of their diet. Third, most previous studies have considered only dietary patterns or fast food consumption, but we investigated the participants' dietary patterns separate from the fast food items they ate and used fast food consumption to identify dietary factors associated with obesity in adults, which allowed us to find that fast food might be not solely responsible for obesity.

\section{Conclusions}

In conclusion, we found that fast food consumption was not directly associated with obesity, whereas the "Meat and alcohol" pattern had independent associations with overweight/obesity and central obesity independent of fast food. Even if fast food is high in calories, the unhealthy dietary pattern has a stronger correlation with overweight/obesity and central obesity than fast food consumption on its own. Our results indicate that more attention should be paid to dietary patterns to prevent and manage overweight/obesity and central obesity among adults in South Korea.

Supplementary Materials: The following are available online at http://www.mdpi.com/2072-6643/11/11/2740/s1, Table S1. Mean of food group intake according to dietary pattern minus any fast food items consumed among adults: KNHNES 10-14.

Author Contributions: The authors' responsibilities were as follows-J.C. and H.L. (Hyunjung Lim) conceived and designed research; D.-Y.K. and H.L. (Hansongyi Lee) collected, analyzed, interpreted the data; D.-Y.K. wrote the manuscript; A.A., J.C., and H.L. (Hyunjung Lim) interpreted the data and reviewed the manuscript; J.C. and H.L. (Hyunjung Lim) had primary responsibility for final content. All authors read and approved the final manuscript. Neither author declared any competing financial interests or conflicts of interests.

Acknowledgments: This study was provided with data from the Korea Centers for Disease Control and Prevention, Republic of Korea.

Conflicts of Interest: The authors declare no conflict of interest.

\section{Abbreviation:}

KNHANES Korea National Health and Nutrition Examination Survey

SSBs Sugar-sweetened beverages

BMI Body mass index

FF Fast foo

OR Odds ratio

CI Confidence interval

\section{References}

1. Field, A.E.; Coakley, E.H.; Must, A.; Spadano, J.L.; Laird, N.; Dietz, W.H.; Rimm, E.; Colditz, G.A. Impact of overweight on the risk of developing common chronic diseases during a 10-year period. Arch. Intern. Med. 2001, 161, 1581-1586. [CrossRef] [PubMed]

2. NCD-RisC. Trends in adult body-mass index in 200 countries from 1975 to 2014: A pooled analysis of 1698 population-based measurement studies with 19.2 million participants. Lancet 2016, 387, 1377-1396. [CrossRef]

3. Asia Pacific Cohort Studies Collaboration. The burden of overweight and obesity in the Asia-Pacific region. Obes. Rev. 2007, 8, 191-196.

4. Popkin, B.M. Global nutrition dynamics: The world is shifting rapidly toward a diet linked with noncommunicable diseases. Am. J. Clin. Nutr. 2006, 84, 289-298. [CrossRef] [PubMed] 
5. Singh, P.N.; Arthur, K.N.; Orlich, M.J.; James, W.; Purty, A.; Job, J.S.; Rajaram, S.; Sabate, J. Global epidemiology of obesity, vegetarian dietary patterns, and noncommunicable disease in Asian Indians. Am. J. Clin. Nutr. 2014, 100, 359s-364s. [CrossRef] [PubMed]

6. Lim, H.; Lee, H.J.; Choue, R.; Wang, Y. Trends in Fast food and Sugar-Sweetened Beverage Consumption and Their Association with Social Environmental Status in South Korea. J. Acad. Nutr. Diet. 2018, 118, 1228-1236. [CrossRef] [PubMed]

7. Zhao, Y.; Wang, L.; Xue, H.; Wang, H.; Wang, Y. Fast food consumption and its associations with obesity and hypertension among children: Results from the baseline data of the Childhood Obesity Study in China Mega-cities. BMC Public Health 2017, 17, 933. [CrossRef] [PubMed]

8. Prentice, A.M.; Jebb, S.A. Fast foods, energy density and obesity: A possible mechanistic link. Obes. Rev. 2003, 4, 187-194. [CrossRef] [PubMed]

9. Pereira, M.A.; Kartashov, A.I.; Ebbeling, C.B.; Van Horn, L.; Slattery, M.L.; Jacobs, D.R., Jr.; Ludwig, D.S. Fast food habits, weight gain, and insulin resistance (the CARDIA study): 15-year prospective analysis. Lancet 2005, 365, 36-42. [CrossRef]

10. Poti, J.M.; Duffey, K.J.; Popkin, B.M. The association of fast food consumption with poor dietary outcomes and obesity among children: Is it the fast food or the remainder of the diet? Am. J. Clin. Nutr. 2014, 99, 162-171. [CrossRef] [PubMed]

11. Duffey, K.J.; Gordon-Larsen, P.; Jacobs, D.R., Jr.; Williams, O.D.; Popkin, B.M. Differential associations of fast food and restaurant food consumption with 3-y change in body mass index: The Coronary Artery Risk Development in Young Adults Study. Am. J. Clin. Nutr. 2007, 85, 201-208. [CrossRef] [PubMed]

12. Duffey, K.J.; Gordon-Larsen, P.; Steffen, L.M.; Jacobs, D.R., Jr.; Popkin, B.M. Regular consumption from fast food establishments relative to other restaurants is differentially associated with metabolic outcomes in young adults. J. Nutr. 2009, 139, 2113-2118. [CrossRef] [PubMed]

13. Xue, H.; Wu, Y.; Wang, X.; Wang, Y. Time Trends in Fast Food Consumption and Its Association with Obesity among Children in China. PLoS ONE 2016, 11, e0151141. [CrossRef] [PubMed]

14. Mazidi, M.; Speakman, J.R. Higher densities of fast food and full-service restaurants are not associated with obesity prevalence. Am. J. Clin. Nutr. 2017, 106, 603-613. [CrossRef] [PubMed]

15. Burgoine, T.; Forouhi, N.G.; Griffin, S.J.; Brage, S.; Wareham, N.J.; Monsivais, P. Does neighborhood fast food outlet exposure amplify inequalities in diet and obesity? A cross-sectional study. Am. J. Clin. Nutr. 2016, 103, 1540-1547. [CrossRef] [PubMed]

16. Zhang, X.; van der Lans, I.; Dagevos, H. Impacts of fast food and the food retail environment on overweight and obesity in China: A multilevel latent class cluster approach. Public Health Nutr. 2012, 15, 88-96. [CrossRef] [PubMed]

17. Shin, H.J.; Cho, E.; Lee, H.J.; Fung, T.T.; Rimm, E.; Rosner, B.; Manson, J.E.; Wheelan, K.; Hu, F.B. Instant noodle intake and dietary patterns are associated with distinct cardiometabolic risk factors in Korea. J. Nutr. 2014, 144, 1247-1255. [CrossRef] [PubMed]

18. Kim, J.; Jo, I.; Joung, H. A rice-based traditional dietary pattern is associated with obesity in Korean adults. J. Acad. Nutr. Diet. 2012, 112, 246-253. [CrossRef] [PubMed]

19. Kweon, S.; Kim, Y.; Jang, M.-j.; Kim, Y.; Kim, K.; Choi, S.; Chun, C.; Khang, Y.-H.; Oh, K. Data resource profile: The Korea national health and nutrition examination survey (KNHANES). Int. J. Epidemiol. 2014, 43, 69-77. [CrossRef] [PubMed]

20. Willett, W. Issues in analysis and presentation of dietary data. Nutr. Epidemiol. 1998, 321-346.

21. World Health Organization. The Asia-Pacific Perspective: Redefining Obesity and Its Treatment; Health Communication Australia: Melbourne, VIC, Australia, 2000.

22. Rural Resources Development Institute. Food Composition Table; 7th revision; Rural Resources Development Institute: Suwon, Korea, 2006. Available online: http://203.241.55.164/ezpdf/customLayoutNew3.jsp? contentId=2F2F493A2F2 F646C5F696D6167652F494D472F30322F2F30303030303030303536333342F534552564 943452F3030303030303030353633345F30312E504446 (accessed on 1 April 2018).

23. National Academy of Agricultural Sciences. Food Composition Table; 8th revision; National Academy of Agricultural Sciences: Suwon, Korea, 2011. Available online: http: //203.241.55.164/ezpdf/customLayoutNew3.jsp?contentId=2F2F493A2F2F646C5F696D6167652F49 4D472F30332F2F30303030303030313333034372F534552564943452F3030303030303031333034375F30312E504446 (accessed on 1 April 2018). 
24. Ministry of Health \& Welfare, The Korean Nutrition Society. Dietary Reference Intakes for Koreans 2015; Ministry of Health \& Welfare, The Korean Nutrition Society: Seoul, Korea, 2015. Available online: http://www.kns.or.kr/FileRoom/FileRoom_view.asp?idx=79\&BoardID=Kdr (accessed on 1 June 2018).

25. Ebbeling, C.B. Sugar-sweetened beverages and body weight. Curr. Opin. Lipidol. 2014, 25, 1-7. [CrossRef] [PubMed]

26. Mary, S.A.L.; Sivagami, A.; Rani, M.U. Cluster validity measures dynamic clustering algorithms. ARPN J. Eng. Appl. Sci. 2015, 10, 4009-4012.

27. International Physical Activity Questionnaire. IPAQ Scoring Protocol. Available online: https://sites.google. $\mathrm{com} /$ site/theipaq/scoring-protocol (accessed on 17 November 2005).

28. Hruby, A.; Manson, J.E.; Qi, L.; Malik, V.S.; Rimm, E.B.; Sun, Q.; Willett, W.C.; Hu, F.B. Determinants and consequences of obesity. Am. J. Public Health 2016, 106, 1656-1662. [CrossRef] [PubMed]

29. Paeratakul, S.; Ferdinand, D.P.; Champagne, C.M.; Ryan, D.H.; Bray, G.A. Fast food consumption among US adults and children: Dietary and nutrient intake profile. J. Am. Diet. Assoc. 2003, 103, 1332-1338. [CrossRef]

30. Cho, Y.A.; Shin, A.; Kim, J. Dietary patterns are associated with body mass index in a Korean population. J. Am. Diet. Assoc. 2011, 111, 1182-1186. [CrossRef] [PubMed]

31. Kang, Y.; Kim, J. Gender difference on the association between dietary patterns and metabolic syndrome in Korean population. Eur. J. Nutr. 2016, 55, 2321-2330. [CrossRef] [PubMed]

32. Song, Y.; Joung, H. A traditional Korean dietary pattern and metabolic syndrome abnormalities. Nutr. Metab. Cardiovasc. Dis. 2012, 22, 456-462. [CrossRef] [PubMed]

33. Ahluwalia, N.; Dwyer, J.; Terry, A.; Moshfegh, A.; Johnson, C. Update on NHANES dietary data: Focus on collection, release, analytical considerations, and uses to inform public policy. Adv. Nutr. 2016, 7, 121-134. [CrossRef] [PubMed]

(C) 2019 by the authors. Licensee MDPI, Basel, Switzerland. This article is an open access article distributed under the terms and conditions of the Creative Commons Attribution (CC BY) license (http://creativecommons.org/licenses/by/4.0/). 